\title{
Distinct Parietal and Temporal Connectivity Profiles of Ventrolateral Frontal Areas Involved in Language Production
}

\author{
Daniel S. Margulies ${ }^{1}$ and Michael Petrides ${ }^{2}$ \\ ${ }^{1}$ Max Planck Research Group: Neuroanatomy \& Connectivity, Max Planck Institute for Human Cognitive and Brain Sciences, 04103, Leipzig, Germany, and \\ ${ }^{2}$ Cognitive Neuroscience Unit, Montreal Neurological Institute, McGill University, Montreal, Quebec, Canada H3A 2B4
}

Broca's region, which in the language-dominant hemisphere of the human brain plays a major role in language production, includes two distinct cytoarchitectonic areas: 44 and 45 . The unique connectivity patterns of these two areas have not been well established. In a resting-state functional connectivity study, we tested predictions about these areas from invasive tract-tracing studies of the connectivity of their homologs in the macaque monkey. We demonstrated their distinct connectivity profiles as well as their differences from the caudally adjacent ventral parts of the premotor cortex and the primary motor cortical region that represent the orofacial musculature. Area 45 is strongly connected with the superior temporal sulcus and the cortex on the adjacent superior and middle temporal gyri. In the parietal region, area 45 is connected with the angular gyrus, whereas area 44 is connected with the supramarginal gyrus. The primary motor cortical region in the caudal precentral gyrus is not connected with the posterior parietal region, which lies outside the confines of the postcentral gyrus, whereas the ventrorostral premotor cortical area 6VR, in the most anterior part of the precentral gyrus, has strong connections with the rostral supramarginal gyrus. Thus, area 44, which has stronger connections to the posterior supramarginal gyrus, can be distinguished from both the adjacent area 6VR and area 45 . These findings provide a major improvement in understanding the connectivity of the areas in the ventrolateral frontal region that are involved in language production.

\section{Introduction}

The traditional view has been that Broca's region, the anterior language region of the human brain composed of the distinct cytoarchitectonic areas 44 and 45 , is primarily connected with the posterior part of the superior temporal gyrus (Geschwind, 1970). The recent discovery of the cytoarchitectonic homologs of areas 44 and 45 in the macaque monkey (Petrides and Pandya, 1994, 2002; Petrides et al., 2005) made it possible to examine their precise anatomical connectivity using invasive tract tracers (Petrides and Pandya, 2009). The macaque monkey studies demonstrated highly distinct connectivity profiles for areas 44 and 45, as well as the adjacent ventrorostral premotor area 6VR that occupies the most anterior part of the precentral gyrus, with the different areas of the inferior parietal and lateral temporal cortex (Fig. 1), suggesting that this may also be the case for the human brain.

The relevance of the macaque research has been supported by tractography studies of the human brain with diffusion magnetic

\footnotetext{
Received May 26, 2013; revised July 26, 2013; accepted Aug. 15, 2013.

Author contributions: D.S.M. and M.P. designed research; D.S.M. and M.P. performed research; D.S.M. analyzed data; D.S.M. and M.P. wrote the paper.

We thank Ziad Saad for contributing analytic tools.

The authors declare no competing financial interests.

Correspondence should be addressed to Daniel S. Margulies, Max Planck Research Group: Neuroanatomy \&

Connectivity, Max Planck Institute for Human Cognitive and Brain Sciences, 04103, Leipzig, Germany. E-mail: margulies@cbs.mpg.de.

DOI:10.1523/JNEUROSCI.2259-13.2013

Copyright $\odot 2013$ the authors $\quad 0270-6474 / 13 / 3316846-07 \$ 15.00 / 0$
}

resonance imaging (MRI), which have also provided evidence for axonal connections between the inferior parietal and lateral temporal regions and the ventrolateral frontal region (Catani et al., 2005; Croxson et al., 2005; Anwander et al., 2007; Frey et al., 2008; Morgan et al., 2009). However, the specific areas of origin of axonal connections in the enormous expanse of cortex that are the parietal and temporal lobes and their termination points in ventrolateral areas 44 and 45 (Broca's region), the premotor area $6 \mathrm{VR}$, and the primary motor area 4 cannot be unambiguously established using diffusion MRI-based approaches (JohansenBerg and Behrens, 2006; Jones, 2008; Roebroeck et al., 2008).

A resting-state functional connectivity analysis in humans provided preliminary evidence that the connectivity profiles of these different areas of the ventrolateral frontal region are similar to those established in the macaque monkey (Kelly et al., 2010). However, the distinctions between the connectivity profiles of areas 44 and 45 within the parietal and temporal regions, clearly established in the monkey, were difficult to discern in that grouplevel analysis. It is important to note that, although area 44 is located primarily on the pars opercularis and area 45 on the pars triangularis of the inferior frontal gyrus, the considerable variability in the morphology of these two parts of the inferior frontal gyrus across individual brains makes it difficult to establish unambiguously their connectivity at the group level.

The aim of the present resting-state functional connectivity study in the human brain was to increase anatomical precision in characterizing the cortical connectivity patterns of these ventrolateral frontal areas by focusing on surface-based individual-level 

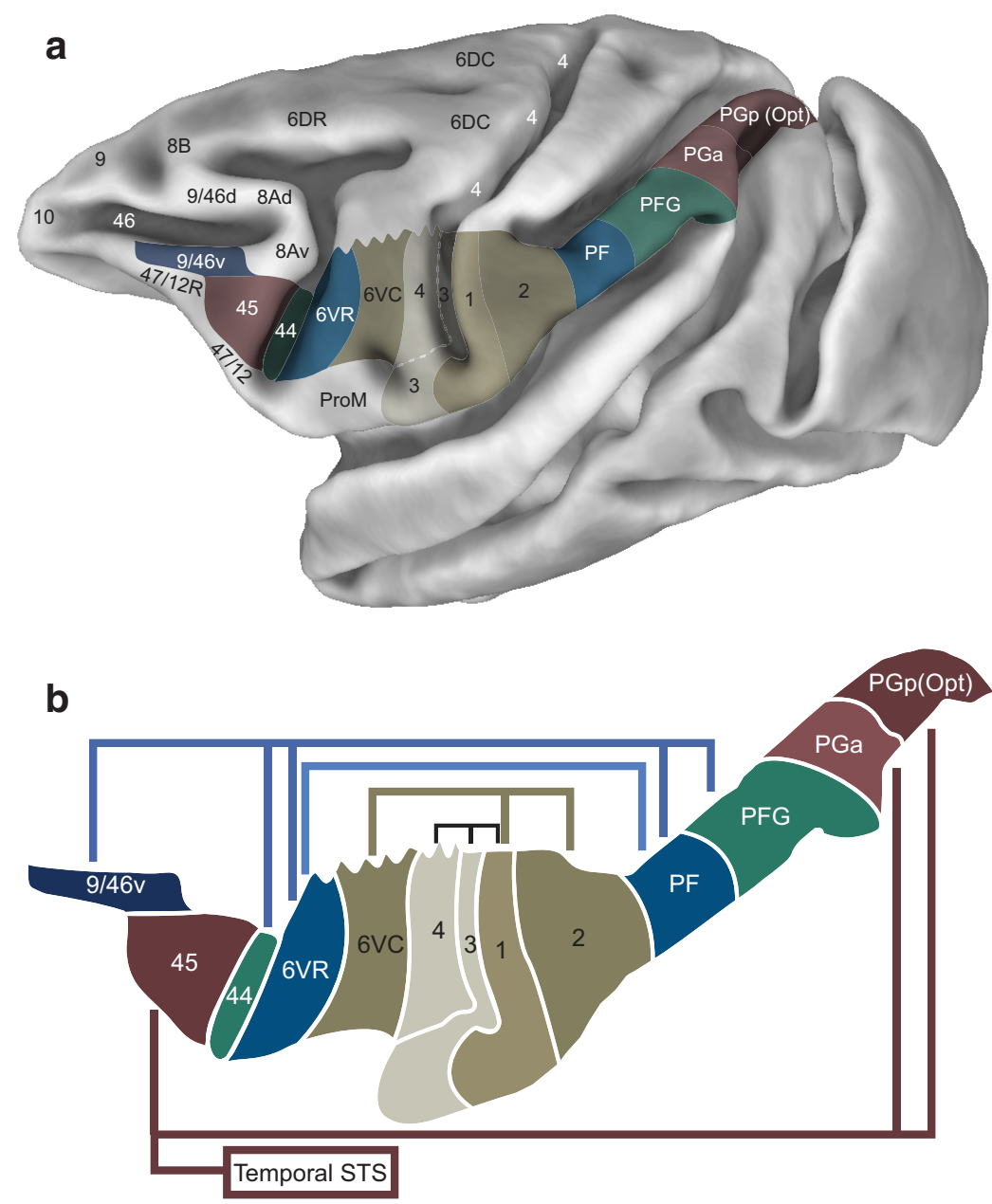

Figure 1. a, Schematic diagram of the architectonic areas of interest along the ventrolateral frontal and inferior parietal cortex in the macaque monkey with anatomical connections indicated $(\boldsymbol{b})$. Note that adjacent areas are always interconnected. R, Rostral; VR, ventrorostral; VC, ventrocaudal; DC, dorsocaudal; $d$, dorsal; v, ventral; a, anterior; p, posterior; STS, superior temporal sulcus; ProM, proisocortical motor area.

analysis. Instead of attempting to establish the boundaries between the areas of interest, namely areas $6 \mathrm{VR}, 44$, and 45 , and to label all their voxels, we placed a single seed region in the estimated center of each area, as well as in the primary motor cortical area 4 . We used $1 \mathrm{~h}$ of resting-state functional MRI (fMRI) data acquired across four scanning sessions from a small group of individuals $(n=6)$ to improve the anatomical specificity of the individual-level connectivity analyses.

\section{Materials and Methods}

Although the areas investigated here each have characteristic topographical landmarks, there is considerable anatomical variation in those landmarks across individual brains. Because areas 44 and 45 lie next to each other, traditional group-level analyses inevitably include a large proportion of misclassified voxels that limit the specificity of the connectivity results. We thus conducted individual-level functional connectivity analyses on a small number of individual datasets. These were of individuals who had undergone multiple resting-state fMRI scans to increase significantly the signal-to-noise ratio. Although it would also have been possible to acquire a single scan of composite length, the separate scan sessions help to minimize motion artifacts that would be attributable to excessively long scans. In addition, to avoid cross-sulcal signal contamination, functional data were not spatially smoothed in the volumetric space but only along the cortical surface. It was expected that such an approach might result in a more precise unveiling of the distinct connectivity signatures that may be partly masked by the problems inherent in group-level studies. Only after determining the individual-level networks from each seed region was a group-level analysis conducted, thus avoiding problems of the results being influenced by anatomical variability in the initial seed location.

Data acquisition. fMRI data were acquired from six healthy, right-handed (as assessed using the Edinburgh Handedness Inventory; Oldfield, 1971) adult participants (25.3 \pm 3.9 years old; three females and three males) who gave informed consent. All protocols were approved by the local ethics board of the Max Planck Institute for Human Cognitive and Brain Sciences in Leipzig, Germany. Restingstate fMRI data were published previously by Taubert et al. (2011).

Four datasets were acquired at 2 week intervals on a Siemens Magnetom Tim Trio 3 Tesla scanner equipped with a 32-channel head coil. The resting-state fMRI data were acquired with the following parameters while the participants were asked to fixate on a crosshair and remain still: 400 whole-brain volumes; acquisition matrix, $64 \times 64$; slice thickness, $3 \mathrm{~mm}$ ( $1 \mathrm{~mm}$ gap); voxel dimensions, $3 \times 3 \times 4 \mathrm{~mm}$; 34 slices; TR, $2300 \mathrm{~ms}$; TE, $30 \mathrm{~ms}$; flip angle, $90^{\circ}$; bandwidth, $1825 \mathrm{~Hz}$; interleaved ascending slice acquisition order. The acquisition time for each resting-state scan was $15.3 \mathrm{~min}$. In addition, four T1-weighted anatomical MPRAGE scans were acquired for each individual at 2 week intervals during the same scanning session with the following parameters: TR, $1300 \mathrm{~s}$; TE, $3.46 \mathrm{~ms}$; flip angle, $10^{\circ}$; FOV , $256 \times 240 \mathrm{~mm}$; 176 sagittal slices; voxel size, $1 \times 1 \times 1.5 \mathrm{~mm}$.

Preprocessing. Preprocessing of the functional data in the volume space was conducted using a pipeline (scripts available at https:// github.com/NeuroanatomyAndConnectivity/ vlpfc_preprocessing_scripts) including commands from AFNI (for Automated Functional Neuro-Imaging; afni.nimh.nih.gov), FSL (for FMRIB Software Library; www.fmrib.ox.ac.uk/fsl/), and FreeSurfer (https:// surfer.nmr.mgh.harvard.edu). The pipeline consisted of the following main preprocessing steps: (1) dropping of first four volumes (3dcalc); (2) slicetiming correction for interleaved ascending slice acquisition (3dTshift); (3) motion correction (3dvolreg); (4) skull stripping (3dAutomask); (5) bandpass filtering between 0.01 and $0.1 \mathrm{~Hz}$ (3dFourier); and (6) linear and quadratic detrending (3dDetrend). Importantly, to avoid cross-sulcal signal contamination, functional data were not spatially smoothed in the volumetric space.

All four MPRAGE anatomical scans were processed together using the standard FreeSurfer cortical surface extraction pipeline (recon-all). Functional and anatomical data were then coregistered (bbregister). The anatomical segmentation from the FreeSurfer output was used to create regions of interest (ROIs) for the ventricles (CSF) and white matter. The average time series from these ROIs and the six motion parameters were then included in a general linear model to extract variance attributable to non-neuronal signal (using film_gls from FSL). The resulting residuals were then prepared for additional surface-based analyses.

The AFNI command $3 d$ Vol2Surf was used to extract the residuals of the functional data onto the left and right cortical surfaces by taking the average value at each node from 15 divisions between the pial and gray/white matter boundary. Smoothing was then conducted on the cortical surface using the HEAT_07 algorithm with an FWHM of 6 mm (SurfSmooth).

For each individual, the four functional runs were prepared for interactive functional connectivity analysis using InstaCorr from AFNI. The 




Figure 2. a, Color-coded schematic of the rostrocaudal arrangement of the ventrolateral and inferior parietal cortical areas of interest. Below is a schematic of the morphology of the ROI, with location of seed regions and anatomical labels: vr, vertical ramus; hr, horizontal ramus; cs, central sulcus; ds, diagonal sulcus; PrG, precentral gyrus; half, horizontal anterior limb of the Sylvian fissure; IFG, inferior frontal gyrus; ifs, inferior frontal sulcus; iprs, inferior precentral; Op, pars opercularis; Tr, pars triangularis; ts, triangular sulcus. $\boldsymbol{b}$, Group level; peak shown as white dots on the inflated right $(\mathrm{RH})$ and left $(\mathrm{LH})$ hemisphere connectivity maps. $\boldsymbol{c}$, Individual-level functional connectivity results from six individuals, color coded as in $\boldsymbol{a}$. Note that, in all cases, there was connectivity with the corresponding homotopic area in the contralateral hemisphere.

advantage of the InstaCorr software for this analysis was the ability to select a seed region and to immediately display its functional connectivity. The four runs were combined using $3 d$ SetupGroupInCorr on the individual cortical surfaces, which enabled cross-session, interactive functional connectivity analysis on the individual level.

Functional connectivity analyses. Functional connectivity was analyzed on the cortical surface using InstaCorr (AFNI) on the cortical surface [SUMA (for Surface Mapper)] using 3dGroupInCorr. This command serves to compute the Fisher's $r$-to-z transform of the correlation values for each run, then to average across them, and finally output the Fisher's $z$-to- $r$ transformed averaged correlation values. Because the four runs were acquired independently from each other, averaging the transformed correlation values was preferable to concatenating the runs, which would have rather required normalization procedures. 3dGroupInCorr facilitated this procedure.

Seed regions were selected based on single nodes (specific seed locations described in Results and Fig. 2a), and mean correlation maps were visualized. For each of the four seed regions, the corresponding statistical map was saved for subsequent cluster-corrected thresholding. Permutation-based cluster-level thresholding was conducted using the AFNI surface-based $\alpha$ probability simulation for the data presented in Figure 2, $b$ and $c$ [nodewise threshold, $r>0.283(p<0.005)$; cluster-level threshold, $p<0.05]$. Because of differences in the cortical topography across individuals, the precise minimum surface area required for cluster-level significance at $p<0.05$ varied from 139 to $332 \mathrm{~mm}^{2}$. For subjects 3 and 5 (Fig. $3 c$ ), a higher nodewise threshold of $p<0.001$ was required for visualizing the results because of the spatially extensive suprathreshold results for these two individuals at $p<0.005$ (cluster-level significance remained at $p<0.05$, resulting in a minimum surface area of 69 and $68 \mathrm{~mm}^{2}$ ). This difference in individual-level threshold did not affect the group-level thresholding.
Group-level analyses were then conducted for each seed region (see Results and Fig. $2 b$ ). Individual surfaces were resampled to consistent group-level nodes using MapIcosahedron with 100 edge divides for linear icosahedron tessellation. Individual-level unthresholded functional connectivity maps were then resampled using SurfToSurf. Statistical maps for each seed region were Fisher's $r$-to- $z$ transformed and averaged across the six participants. Resultant statistical maps were then thresholded using the same permutation-based cluster-level correction procedure described above with the nodewise threshold of $p<0.005$ and clusterlevel threshold of $p<0.05$ (minimum surface area, $150 \mathrm{~mm}^{2}$ ).

Results were visualized by combining the four thresholded maps on a single surface map. For the individuals (Fig. $2 c$ ), the individual-level pial surfaces were inflated to facilitate viewing within the sulci. White dots indicated the peak of the statistical map and represent the location of the node used as the seed region. Group-level results (Fig. $2 b$ ) were presented on both pial and inflated surfaces to emphasize the relationship of the network anatomy to the cortical topography (pial), as well as the respective position of the networks with respect to one another (inflated).

\section{Results}

The results are presented in a caudal-to-rostral direction, starting with the primary motor cortical area 4 and moving anterior to prefrontal area 45. Because of the considerable individual variability across brains, the seed was selected on the basis of the local morphology and known relationships of the local morphology to cytoarchitectonic areas. Thus, rather than present mean volume locations in MNI standard stereotaxic space, which would be of limited value given the cortical variability of the location of the areas with respect to the sulci and gyri, we provide a description of how the sulci were used to select the areas of interest. 


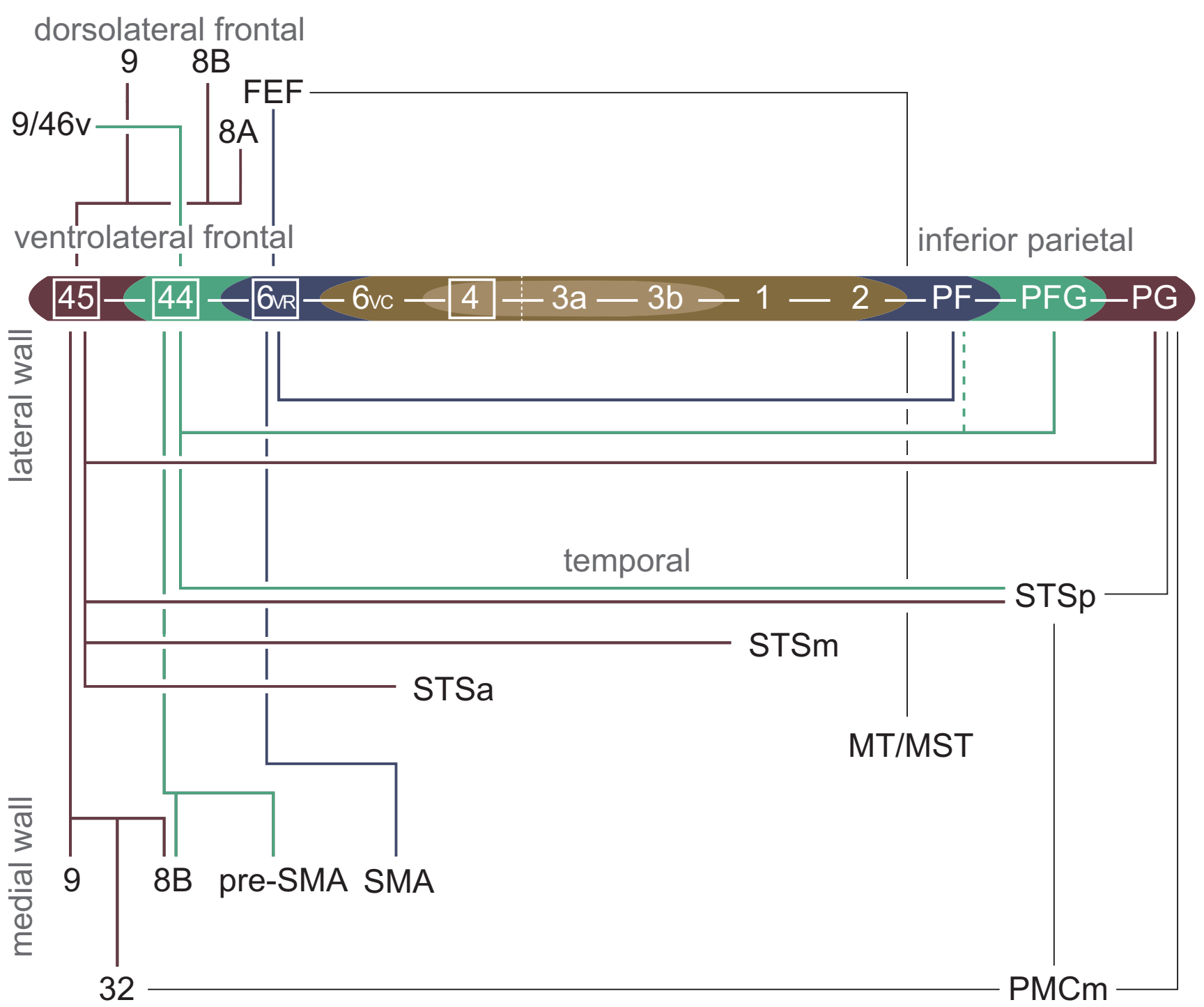

Figure 3. Summary diagram of the connectivity profiles of the ventrolateral frontal areas of interest. Terms in gray refer to cortical lobes, and terms in black refer to specific cortical areas: STS, superior temporal sulcus; PMCm, middle posteromedial cortex; a, anterior; m, middle; $p$, posterior.

\section{Area 4}

The seed for the primary motor cortical area 4 was placed in the anterior bank of the central sulcus because we know that the ventral part of this area, which represents the orofacial musculature (Penfield and Boldrey, 1937), is virtually confined there (Brodmann, 1909; Rademacher et al., 2001). The resulting functional connectivity extended along the precentral and postcentral gyri, in some cases restricted to the ventral part (Fig. $2 c$, case 4 ), the adjacent central opercular region (i.e., secondary somatosensory cortex), and the insular cortex. On the medial surface, connectivity was restricted to the motor region of the paracentral lobule and the adjacent supplementary motor area (SMA); however, functional connectivity was also observed to portions of primary and secondary visual cortex. Because no known direct anatomical connections exist, such correlation may be driven by thalamic inputs. The connectivity to the right hemisphere was confined to topographically homotopic areas (Fig. 2b, RH). This overall connectivity pattern is typical of the core somatomotor region (Vogt and Pandya, 1978; Dum and Strick, 2002).

\section{Area 6VR}

The seed for the ventral part of the rostral premotor area 6VR was placed in the anteriormost part of the precentral gyrus, extending into the anterior bank of the inferior precentral gyrus. In other words, the seed was placed in the edge of the ventral precentral region and into the posterior bank of the inferior precentral sulcus. Functional connectivity was observed with the rostralmost portion of inferior parietal lobule, namely the rostral part of the supramarginal gyrus (area PF), but not with the postcentral gyrus (Figs. 1, 2), clearly distinguishing rostral area 6 from primary motor cortical area 4 . In our previous group-level study (Kelly et al., 2010), although we could demonstrate that area 6VR showed connectivity with area PF, we could not discriminate it from primary motor cortex because of extensive connectivity with the core somatomotor region. Connectivity to the right hemisphere on the group level was to the ventral homologs and the dorsal premotor area. In addition, correlated activity was observed at the boundary of the occipitotemporal region in relation to the ventral anterior occipital sulcus, a sulcus also referred to as the ascending limb of the inferior temporal sulcus (Petrides, 2012). 
Functional neuroimaging studies have identified this region as the motion processing occipitotemporal region that includes areas MT/MST (Dumoulin et al., 2000). In the macaque monkey, there is strong evidence that this region is anatomically linked to the frontal eye field (FEF) (Ungerleider and Desimone, 1986), which, in turn, is linked to the dorsal part of 6VR. Therefore, we consider that this connectivity may have been driven indirectly by the connectivity of 6VR with the FEF (Fig. 3).

\section{Area 44}

The seed for area 44 was placed in the center of the pars opercularis, following cytoarchitectonic studies of its location (Amunts et al., 1999), to avoid the ambiguous borders with area 6VR along the inferior precentral sulcus and with area 45 along the ascending ramus of the Sylvian fissure. The pars opercularis is defined as that part of the inferior frontal gyrus that lies ventral to the inferior frontal sulcus and anterior to the inferior precentral sulcus. Rostrally, it is demarcated from the pars triangularis by the vertical ramus of the Sylvian fissure. Area 44 exhibited no connectivity with the core somatomotor region along the central sulcus, its connectivity being with the supramarginal gyrus (areas PF and PFG) as a whole, but most strongly with the caudal part of the supramarginal gyrus (area PFG). This feature clearly differentiated it from the primary motor cortical region that was connected with the postcentral gyrus but not with the supramarginal gyrus. Although area 6VR shared some aspects of connectivity with area 44 , the latter area had stronger connectivity with the caudal part of the supramarginal gyrus, namely area PFG. This finding is consistent with predictions from the macaque monkey data (Petrides and Pandya, 2009). The connectivity of area 44 extended to the nearby caudalmost part of the superior temporal gyrus and the adjacent section of the superior temporal sulcus. The connectivity with the more anterior part of the lateral temporal cortex was weak, as would be expected from the macaque monkey studies (Petrides and Pandya, 2009). On the lateral frontal lobe, there was connectivity with the part of the middle frontal gyrus that lies immediately dorsal to the pars triangularis. This part of the middle frontal gyrus is occupied by a special type of cortex known as area 9/46v (Petrides and Pandya, 1994), which has been shown, in the monkey, to be strongly connected with the homologs of areas 44 and PFG. Contralateral connectivity on the group level was specific to the pars opercularis homolog. On the medial surface, the connectivity was with the anterior part of medial area 6 (SMA) at its border with pre-SMA and the cortex adjacent to the cingulate sulcus.

\section{Area 45}

The seed for area 45 was placed in the pars triangularis of the inferior frontal gyrus, following architectonic data about its location (Amunts et al., 1999). We identified the pars triagularis as the part of the inferior frontal gyrus that lies between the vertical and horizontal rami of the Sylvian fissure and that is capped dorsally by the inferior frontal sulcus and anteriorly by the pretriangular sulcus (Petrides, 2012). The cortex of the pars triangularis is occupied by area 45 . Anterodorsally, area 45 is succeeded by area $9 / 46 \mathrm{v}$ when we exit the pars triangularis close to the anteriormost part of the inferior frontal sulcus (Petrides and Pandya, 2002). We placed the seed in the center of the region of the pars triangularis but ventral enough to avoid any overlap with voxels belonging to either area $9 / 46 \mathrm{v}$ or area 44 .

The connectivity of area 45 was strikingly different from that of area 44 . Area 45 had strong connections with the angular gyrus (area PG), which lies between the branches of the caudal superior temporal sulcus. Importantly, within the lateral temporal lobe, area 45 exhibited significant connectivity along the entire extent of the superior temporal sulcus and the immediately adjacent parts of the superior and middle temporal gyri. On the medial surface of the hemisphere, the connectivity of area 45 was with cortex anterior to the SMA, involving the pre-SMA region and areas 8 and 9 on the medial surface of the superior frontal gyrus, as well as the posteriorly located central precuneal region [area PGm, located in the middle posterior medial cortex (PMCm); Margulies et al., 2009]. In the dorsolateral frontal lobe, area 45 had connectivity with cortical regions corresponding to areas 8 and 9. In the right hemisphere, connectivity was to the pars triangularis homolog as well as the superior temporal sulcus of the temporal lobe.

\section{Discussion}

The present results demonstrated, in the human brain, the unique connectivity profiles of the two areas that comprise Broca's region, namely areas 44 and 45 , and also succeeded in differentiating the connectivity of the primary motor cortex (area 4) (which is found in the posterior part of the precentral gyrus along the central sulcus) from the rostral premotor area (area 6VR) at the most anterior part of the precentral gyrus.

Area 45 could be distinguished from all other areas of interest by its strong and unique connectivity with the cortex within the superior temporal sulcus and the immediately adjacent superior and middle temporal gyri. Within the parietal region, area 45 was uniquely connected with the cortex of the angular gyrus, which is found around the caudal branches of the superior temporal sulcus as they invade the caudal inferior parietal region. In contrast, area 44 was connected primarily with the supramarginal gyrus, particularly to its caudal part.

The ventral part of the precentral gyrus (area 6VR) represents the orofacial musculature as shown by the classic electrical stimulation research of Penfield and colleagues (such as Penfield and Boldrey, 1937) and modern functional neuroimaging research (e.g., Martin et al., 2004). Cytoarchitectonically, the ventral precentral gyrus is divided into the caudal part, which includes the anterior bank of the central sulcus in which area 4 lies and the immediately adjacent caudal area 6 (i.e., 6VC), and the more anterior part in which area 6VR lies and that extends into the posterior bank of the inferior precentral sulcus. Area 6VR on the anteriormost part of the precentral gyrus was strongly linked with the rostral supramarginal gyrus and could be distinguished from the primary motor region, which was connected primarily with the postcentral gyrus (i.e., somatosensory cortex).

Therefore, the results demonstrate that the ventral part of the primary motor cortex, in which the orofacial region is represented (Penfield and Boldrey, 1937; Martin et al., 2004), is linked with adjacent premotor cortex on the precentral gyrus and somatosensory cortex on the postcentral gyrus, but it has no way of communicating directly with Broca's region, except indirectly via the premotor cortex.

These results provided a level of precision in describing the connectivity of the ventrolateral frontal areas that had not been achieved previously and are consistent with predictions from the gold-standard macaque monkey tract-tracing data (Petrides and Pandya, 2009; Fig. 1). This significant advance in understanding the precise connectivity of these regions in the human brain provides a solid foundation for insights into the cortical organization underlying human language capacity. For instance, the distinct connectivity profiles of the two areas that comprise Broca's region, area 45 on the pars triangularis and area 44 on the pars opercularis, predict major functional differences. There is evi- 
dence from functional neuroimaging that the two parts of Broca's region play an important role in verbal fluency, with area 44 appearing to play a more important role in phonological fluency (Heim et al., 2008). In contrast, area 45 is much more involved in active controlled retrieval of information from memory (Petrides et al., 1995; Kostopoulos and Petrides, 2003). Therefore, it is of interest that a recent functional neuroimaging study has provided strong evidence for phonological processing in the left supramarginal gyrus (which is linked with area 44) but not the left angular gyrus (Church et al., 2011). In contrast, the left angular gyrus that is linked with area 45 is involved in semantic processing (Binder et al., 2009) and is also involved in reading (Segal and Petrides, 2013).

Although resting-state functional connectivity can only indicate correlations between areas, there is strong evidence from macaque monkeys that the connections between area 45 and the lateral temporal cortex are made via a newly discovered fasciculus, the temporo-frontal extreme capsule fasciculus (Petrides and Pandya, 1988, 2009). Diffusion-based tractography has provided evidence for the existence of this pathway also in the human brain (Frey et al., 2008), although it cannot establish its precise origin in the temporal lobe or its terminations in the ventrolateral frontal region. Recent studies combining functional neuroimaging with diffusion MRI have strongly suggested that the temporal to ventrolateral frontal connections via the extreme capsule fasciculus might be the basis of the ventral language stream underlying semantic processing as opposed to the dorsal stream via the arcuate fasciculus underlying phonological aspects of language processing (Saur et al., 2008).

\section{Individual- versus group-level analysis}

Our previous group-level analysis (Kelly et al., 2010) demonstrated that the ventral premotor cortical area 6VR has a strikingly different connectivity profile from the more anteriorly located Broca's region that includes areas 44 and 45: premotor cortex had a strong somatomotor connectivity profile, including the whole precentral and postcentral gyri and rostral supramarginal, whereas Broca's region was linked with cortex of the inferior parietal lobule. However, this group-level analysis (1) failed to separate the premotor cortex from the primary motor cortex (clearly demonstrated in the macaque monkey studies) and (2) could not separate clearly the connectivity profiles of the two areas that comprise Broca's region, although there were indications of distinct patterns. The macaque monkey data based on the tracing of axonal connections between parietal and temporal cortical areas and the homologs of areas 44 and 45 showed strikingly distinct connectivity profiles. We assumed that, because areas 44 and 45 lie adjacent to each other on the pars opercularis and pars triangularis, respectively, and the morphology of these two parts varies considerably in MNI standard space, our attempt in the group-level analysis to outline and label all the voxels that constitute these two areas must have inevitably included many misclassified voxels at the borders of these areas, limiting the specificity of the demonstrated connections.

The axonal connection studies in the macaque monkey demonstrated unambiguously that area 44 has connections primarily with area PFG, whereas area 45 has connections with area PG (Petrides and Pandya, 2009). The individual-level results presented here succeeded in clearly demonstrating in the human brain the strong connectivity of area 44 with PFG (caudal supramarginal gyrus) and of area 45 with area PG (angular gyrus).

Furthermore, the macaque monkey studies showed another striking difference between area 45 and area 44 : area 45 has mas- sive connections with the lateral temporal cortex, along the superior temporal sulcus, whereas area 44 has moderate connections with the temporal region and focused on its posterior part. Our previous group-level analyses (Kelly et al., 2010) required ROI analyses to derive a relatively minor difference in connectivity with lateral temporal cortex between areas 45 and 44, but the individual-level analysis demonstrates this difference clearly.

A significant difference in the current method was that, instead of attempting to label all voxels with the inevitable misclassification at the borders of areas, we placed the seed voxel at the heart of each area using available anatomical knowledge of its location. The specificity of these differential results also suggests that the surface-based spatial smoothing and analysis and the coregistration after functional connectivity analysis (which minimizes the confound of intersubject anatomical variability) are significant factors. Notably, no connectivity was observed in the superior temporal gyrus from adjacent regions in the upper bank of the lateral fissure that virtually touch it, in contrast to results from our group-level study (Kelly et al., 2010), again suggesting improved anatomical registration.

\section{Interhemispheric homologs}

Although cytoarchitectonically defined areas 45 and 44 are mostly topographically consistent with the anatomical landmarks of the ventrolateral prefrontal cortex in the left hemisphere, they are more difficult to localize in the right hemisphere. Across all four areas, we observed functionally connected contralateral homologs on the group level that correspond to the precise anatomical locations of these regions (Fig. $2 b, \mathrm{RH}$ ). Although intrinsic functional connectivity has been demonstrated to have substantial interhemispheric homotopy (Stark et al., 2008; Jo et al., 2012), previous clustering results across 1000 datasets demonstrated substantial lower clustering confidence in borders along the ventrolateral frontal cortex - an observation that was even more prominent in the right hemisphere (Yeo et al., 2011).

\section{Distinct medial wall connectivity}

We found previously distinct functional connectivity to areas of the medial wall, organized in a caudal-rostral gradient (Kelly et al., 2010), and diffusion MRI-based tractography also supports this organization (Ford et al., 2010). Although the medial connectivity of area 6VR did not exceed the threshold on the group level, the individual-level patterns demonstrated this caudal-rostral gradient, mirroring the lateral ordering. Even in the absence of significant connectivity from any single area, the basic topological relationships were maintained.

\section{Conclusions}

The current study demonstrates that neuroanatomical questions can potentially be answered with a high degree of precision on the individual level using resting-state fMRI data. These findings support additional use of individual-level analyses in addressing anatomical questions and also demonstrate the feasibility of this approach for clinical applications (e.g., neurosurgical planning), which inevitably require answers at the individual level. For instance, in situations that require the investigator to identify areas 44 and 45, resting-state functional connectivity can be used to separate them through their distinct parietal and temporal connectional fingerprints.

\section{Notes}

Supplemental material for this article, which consists of scripts used for preprocessing of the data, is available at https://github.com/Neuroanatomy 
AndConnectivity/vlpfc_preprocessing_scripts. This material has not been peer reviewed.

\section{References}

Amunts K, Schleicher A, Bürgel U, Mohlberg H, Uylings HB, Zilles K (1999) Broca's region revisited: cytoarchitecture and intersubject variability. J Comp Neurol 412:319-341. CrossRef Medline

Anwander A, Tittgemeyer M, von Cramon DY, Friederici AD, Knösche TR (2007) Connectivity-based parcellation of Broca's area. Cereb Cortex 17: 816-825. CrossRef Medline

Binder JR, Desai RH, Graves WW, Conant LL (2009) Where is the semantic system? A critical review and meta-analysis of 120 functional neuroimaging studies. Cereb Cortex 19:2767-2796. CrossRef Medline

Brodmann K (1909) Vergleichende Lokalisationslehre der Grosshirnrinde in ihren Prinzipien dargestellt auf Grund des Zellenbaues von Dr. K. Brodmann. Leipzig: J. A. Barth.

Catani M, Jones DK, ffytche DH (2005) Perisylvian language networks of the human brain. Ann Neurol 57:8-16. CrossRef Medline

Church JA, Balota DA, Petersen SE, Schlaggar BL (2011) Manipulation of length and lexicality localizes the functional neuroanatomy of phonological processing in adult readers. J Cogn Neurosci 23:1475-1493. CrossRef Medline

Croxson PL, Johansen-Berg H, Behrens TE, Robson MD, Pinsk MA, Gross CG, Richter W, Richter MC, Kastner S, Rushworth MF (2005) Quantitative investigation of connections of the prefrontal cortex in the human and macaque using probabilistic diffusion tractography. J Neurosci 25: 8854-8866. CrossRef Medline

Dum RP, Strick PL (2002) Motor areas in the frontal lobe of the primate. Physiol Behav 77:677-682. CrossRef Medline

Dumoulin SO, Bittar RG, Kabani NJ, Baker CL Jr, Le Goualher G, Bruce Pike G, Evans AC (2000) A new anatomical landmark for reliable identification of human area V5/MT: a quantitative analysis of sulcal patterning. Cereb Cortex 10:454-463. CrossRef Medline

Ford A, McGregor KM, Case K, Crosson B, White KD (2010) Structural connectivity of Broca's area and medial frontal cortex. Neuroimage 52: 1230-1237. CrossRef Medline

Frey S, Campbell JS, Pike GB, Petrides M (2008) Dissociating the human language pathways with high angular resolution diffusion fiber tractography. J Neurosci 28:11435-11444. CrossRef Medline

Geschwind N (1970) The organization of language and the brain. Science 170:940-944. CrossRef Medline

Heim S, Eickhoff SB, Amunts K (2008) Specialisation in Broca's region for semantic, phonological, and syntactic fluency? Neuroimage 40:13621368. CrossRef Medline

Jo HJ, Saad ZS, Gotts SJ, Martin A, Cox RW (2012) Quantifying agreement between anatomical and functional interhemispheric correspondences in the resting brain. PLoS One 7:e48847. CrossRef Medline

Johansen-Berg H, Behrens TE (2006) Just pretty pictures? What diffusion tractography can add in clinical neuroscience. Curr Opin Neurol 19:379385. CrossRef Medline

Jones DK (2008) Studying connections in the living human brain with diffusion MRI. Cortex 44:936-952. CrossRef Medline

Kelly C, Uddin LQ, Shehzad Z, Margulies DS, Castellanos FX, Milham MP, Petrides M (2010) Broca's region: linking human brain functional connectivity data and non-human primate tracing anatomy studies. Eur J Neurosci 32:383-398. CrossRef Medline

Kostopoulos P, Petrides M (2003) The mid-ventrolateral prefrontal cortex: insights into its role in memory retrieval. Eur J Neurosci 17:1489-1497. CrossRef Medline

Margulies DS, Vincent JL, Kelly C, Lohmann G, Uddin LQ, Biswal BB, Villringer A, Castellanos FX, Milham MP, Petrides M (2009) Precuneus shares intrinsic functional architecture in humans and monkeys. Proc Natl Acad Sci U S A 106:20069-20074. CrossRef Medline

Martin RE, MacIntosh BJ, Smith RC, Barr AM, Stevens TK, Gati JS, Menon RS (2004) Cerebral areas processing swallowing and tongue movement are overlapping but distinct: a functional magnetic resonance imaging study. J Neurophysiol 92:2428-2443. CrossRef Medline

Morgan VL, Mishra A, Newton AT, Gore JC, Ding Z (2009) Integrating functional and diffusion magnetic resonance imaging for analysis of structure-function relationship in the human language network. PLoS One 4:e6660. CrossRef Medline

Oldfield RC (1971) The assessment and analysis of handedness: the Edinburgh inventory. Neuropsychologia 9:97-113. CrossRef Medline

Penfield W, Boldrey E (1937) Somatic motor and sensory representation in the cerebral cortex of man as studied by electrical stimulation. Brain 60:389-443. CrossRef

Petrides M (2012) The human cerebral cortex: an MRI atlas of the sulci and gyri in MNI stereotaxic space, Ed 1. Waltham, MA: Elsevier Academic.

Petrides M, Pandya DN (1988) Association fiber pathways to the frontal cortex from the superior temporal region in the rhesus monkey. J Comp Neurol 273:52-66. CrossRef Medline

Petrides M, Pandya DN (1994) Comparative architectonic analysis of the human and the macaque frontal cortex. In: Handbook of neuropsychology (Boller F, Grafman J, eds). Amsterdam: Elsevier Science.

Petrides M, Pandya DN (2002) Comparative cytoarchitectonic analysis of the human and the macaque ventrolateral prefrontal cortex and corticocortical connection patterns in the monkey. Eur J Neurosci 16:291-310. CrossRef Medline

Petrides M, Pandya DN (2009) Distinct parietal and temporal pathways to the homologues of Broca's area in the monkey. PLoS Biol 7:e1000170. CrossRef Medline

Petrides M, Alivisatos B, Evans AC (1995) Functional activation of the human ventrolateral frontal cortex during mnemonic retrieval of verbal information. Proc Natl Acad Sci U S A 92:5803-5807. CrossRef Medline

Petrides M, Cadoret G, Mackey S (2005) Orofacial somatomotor responses in the macaque monkey homologue of Broca's area. Nature 435:1235-1238. CrossRef Medline

Rademacher J, Bürgel U, Geyer S, Schormann T, Schleicher A, Freund HJ, Zilles K (2001) Variability and asymmetry in the human precentral motor system. A cytoarchitectonic and myeloarchitectonic brain mapping study. Brain 124:2232-2258. CrossRef Medline

Roebroeck A, Galuske R, Formisano E, Chiry O, Bratzke H, Ronen I, Kim DS, Goebel R (2008) High-resolution diffusion tensor imaging and tractography of the human optic chiasm at 9.4 T. Neuroimage 39:157-168. CrossRef Medline

Saur D, Kreher BW, Schnell S, Küummerer D, Kellmeyer P, Vry MS, Umarova R, Musso M, Glauche V, Abel S, Huber W, Rijntjes M, Hennig J, Weiller C (2008) Ventral and dorsal pathways for language. Proc Natl Acad Sci U S A 105:18035-18040. CrossRef Medline

Segal E, Petrides M (2013) Functional activation during reading in relation to the sulci of the angular gyrus region. Eur J Neurosci 38:2793-2801. CrossRef Medline

Stark DE, Margulies DS, Shehzad ZE, Reiss P, Kelly AM, Uddin LQ, Gee DG, Roy AK, Banich MT, Castellanos FX, Milham MP (2008) Regional variation in interhemispheric coordination of intrinsic hemodynamic fluctuations. J Neurosci 28:13754-13764. CrossRef Medline

Taubert M, Lohmann G, Margulies DS, Villringer A, Ragert P (2011) Longterm effects of motor training on resting-state networks and underlying brain structure. Neuroimage 57:1492-1498. CrossRef Medline

Ungerleider LG, Desimone R (1986) Cortical connections of visual area MT in the macaque. J Comp Neurol 248:190-222. CrossRef Medline

Vogt BA, Pandya DN (1978) Cortico-cortical connections of somatic sensory cortex (areas 3,1 and 2) in the rhesus monkey. J Comp Neurol 177:179-191. CrossRef Medline

Yeo BT, Krienen FM, Sepulcre J, Sabuncu MR, Lashkari D, Hollinshead M, Roffman JL, Smoller JW, Zöllei L, Polimeni JR, Fischl B, Liu H, Buckner RL (2011) The organization of the human cerebral cortex estimated by intrinsic functional connectivity. J Neurophysiol 106:1125-1165. CrossRef Medline 